\title{
Predictors of In-Hospital Mortality Among Suspected Stroke Patients, Mashhad, Iran: An Application of Autologistic Regression Model
}

Mohammad Taghi Shakeri

Mashhad University of Medical Sciences

Isa Nazar

Mashhad University of Medical Sciences

Azadeh Saki

Mashhad University of Medical Sciences

Razieh Yousefi

Mashhad University of Medical Sciences

Ali Hadianfar ( $\nabla$ hadianfara981@mums.ac.ir)

Mashhad University of Medical Sciences

Payam Sasannezhad

Mashhad University of Medical Sciences

Zahra jafari

Mashhad University of Medical Sciences

\section{Research Article}

Keywords: Suspected stroke, In-hospital mortality, Emergency Medical Services, Autologistic model

Posted Date: March 24th, 2021

DOl: https://doi.org/10.21203/rs.3.rs-337328/v1

License: (c) (i) This work is licensed under a Creative Commons Attribution 4.0 International License.

Read Full License 


\title{
Predictors of in-hospital mortality among suspected stroke patients, Mashhad, Iran: An application of autologistic regression model
}

\author{
Mohammad Taghi Shakeri ${ }^{1}$, Payam Sasannezhad ${ }^{2}$, Isa Nazar ${ }^{3,4}$, Azadeh Saki ${ }^{4}$, ,Razieh Yousefi \\ ${ }^{3,4}$, Ali Hadianfar ${ }^{3,4 *}$, Zahra jafari ${ }^{5}$ \\ ${ }^{1}$ Professor of Biostatistics, Social Determinants of Health Research Center, Mashhad University \\ of Medical Sciences, Mashhad, Iran \\ ${ }^{2}$ Department of Neurology, Mashhad University of Medical Sciences, Mashhad, Iran \\ ${ }^{3}$ Student Research Committee, Mashhad University of Medical Sciences, Mashhad, Iran \\ ${ }^{4}$ Department of Biostatistics, School of Public Health, Mashhad University of Medical Science, \\ Mashhad, Iran \\ ${ }^{5}$ Clinical Research Development Unit, Ghaem Hospital, Mashhad University of Medical \\ Sciences, Mashhad, Iran
}

*Corresponding author: Ali Hadianfar, Department of Biostatistics, School of Public Health, Mashhad University of Medical Science, Mashhad, Iran, Email: hadianfara981@mums.ac.ir

\begin{abstract}
Background: Stroke is the second leading cause of death in adults worldwide. There are remarkable geographical variations in the accessibility to emergency medical services (EMS), and transport delays have been documented worldwide to affect stroke outcomes significantly. Therefore, this study examines whether there are spatial variations in in-hospital mortality among suspected stroke patients transferred by EMS and attempts to determine its related factors using the auto logistic regression model.
\end{abstract}

Methods: In this historical cohort study, suspected stroke patients transferred to Ghaem Hospital of Mashhad by the EMS from April 2018 to March 2019 were included. Using emergency mission IDs, the baseline EMS data were integrated with the follow-up hospital records. The autologistic regression model was applied to examine the possible geographical variations in inhospital mortality and its related factors. All analysis was carried out by SPSS version 16 and R 4.0.0 at the significant level of 0.05 .

Results: 1,222 suspected stroke patients were included in this study, and the in-hospital mortality rate was $14.2 \%$. Overall in-hospital stroke mortality was related to age, accessibility rate of an ambulance, screening time, and length of stay $(\mathrm{p}<0.05)$. After stratifying by sex, we observed that mortality in men was related to age and length of stay, whereas, in women, variables of age, length of stay, accessibility rate of an ambulance, and screening time had a significant effect on in-hospital mortality among suspected stroke patients $(\mathrm{p}<0.05)$.

Conclusion: Our results showed considerable geographical variations in in-hospital stroke mortality in Mashhad neighborhoods. Also, age- and sex-adjusted results from this study highlight the direct association between accessibility rate of an ambulance, screening time and 
length of stay, and in-hospital stroke mortality. The prognosis of in-hospital stroke mortality could be improved by reducing delay time and increasing the EMS access rate.

Keywords: Suspected stroke, In-hospital mortality, Emergency Medical Services, Autologistic model

\section{Introduction}

Stroke is a major health problem worldwide, and its burden on community health has been increasing over the years $(1,2)$. Stroke is the second leading cause of death and disability in adults around the world $(3,4)$. Despite the preventable nature of the disease, two-thirds of all stroke deaths occur in developing countries(5-7). In Iran, studies have reported that the overall in-hospital mortality is $20.5 \%(8,9)$, and in comparison to developed countries, stroke is more common, and the mortality rate is higher $(10,11)$.

Identifying the risk factors for stroke is one of the most important epidemiological aspects of preventing stroke and has significantly reduced its incidence in countries and increased life expectancy (12). One of the most important factors in stroke patients' survival is timely admission and transfer of patients from an Emergency Department to the hospital because stroke needs emergency care medicine (13). For every minute of delay in treating ischemic stroke, a patient loses typically about 1.9 million brain cells (14). Received intravenous thrombolytic therapy within 3 to 4 hours of the onset of symptoms can prevent tissue death and save patients (15). While there are two types of general delays (prehospital and hospital) that affect the timing of stroke treatment, some studies have shown that prehospital delay is the onset of symptoms until the patient arrives at the hospital, which is more important and longer (16). Emergency medical services can be associated with a reduction in prehospital delays in stroke treatment (16). Another important and influential factor of stroke management is the patient's place of residence. Many studies mentioned that neighborhood characteristics and structure might play an important role in spreading or preventing disease (17). In the case of stroke, growing evidence suggests that neighborhood tissue is associated with post-stroke mortality and regional disparities in stroke mortality persist (18-20). For example, people who live in neighborhoods with better neighborhood social ties have a lower risk of death (20). Therefore, it is more appropriate to incorporates spatial components in usual models. 
The logistic regression model is an appropriate model for analyzing binary outcome data. However, typically, spatial autocorrelation is frequently present in disease occurrences and risk factors. It appears that adjacent units in space tend to have more similar values than those distributed randomly. Besag et al. introduced an auto logistic regression model considering spatial autocorrelation, a special type of ordinary logistic model (21). Previous studies usually used an ordinary logistic regression model to determine predictors of in-hospital mortality after stroke, and it seems that there is a lack of evidence to capture the spatial structure of in-hospital mortality among these patients $(9,22,23)$. Our study's primary aim was to evaluate the possible geographical variations of in-hospital mortality and its determinants among suspected stroke patients transferred to Ghaem Hospital of Mashhad in Iran by the EMS using the autologistic regression model.

\section{Methods}

\section{Study area and data sources}

In this historical cohort study, all patients with suspected stroke that EMS transferred to the Neurology ward at Ghaem Hospital in Mashhad from March 2018 to March 2019 were examined. Mashhad is the second-most populous city in Iran with more than 3.00 million inhabitants, based on the 2016 national census of Iran (24). The neurology center of Ghaem educational Hospital of Mashhad University of Medical Sciences (MUMS) is a tertiary neurological referral center in the east of Iran, and a. All neurology emergency care is supplied at this hospital. The data in this study includes two parts: Baseline EMS information (prehospital) and Follow-up information (screening and hospital ward information), which were received from the EMS system database and HIS unit of Ghaem Hospital in Mashhad, respectively. Integrating two datasets was performed by using Emergency mission ID.

\section{Data Analysis}

In-hospital mortality of suspected stroke patients was defined as the outcome variable. Independent variables were delay time $(\mathrm{Sec})$, the response time (minute), transport time (minute), distance to the hospital (km), screening time (hour), length of hospital stay, and hypertension, as well as age, and gender were considered as confounders. Also, the accessibility rate of the ambulance (the number of ambulances per 1 million inhabitants) for each patient was obtained (25). 
Descriptive statistics are used to summarize the basic features of the data. The continuous and qualitative variables are summarized as mean $\pm \mathrm{SD}$ or median (IQR) and frequency (\%). Comparisons mean of quantitative variables performed by independent T-Test, and the association between qualitative variables were assessed using Chi-square test. Local Moran's I Statistic was applied to measure the local spatial autocorrelation and clustering tendencies across neighborhoods. This statistic provides information related to the location of spatial clusters and outliers and the types of spatial correlation (26). We used the autologistic regression model to estimate odds ratios (OR) and 95\% confidence intervals (CIs) of predictors of in-hospital mortality.

The autologistic regression model has been used widely for modeling spatially correlated binary data. Compared to the ordinary logistic model, the autologistic model introduces a spatial autocorrelation term, autocovariate, as shown in the following equations. Autocovariates are weighting coefficients calculated by Euclidean distance in the form of the dependent variable's total weight.

$$
\begin{aligned}
& Y_{i} \sim \text { Binomial }\left(p_{i}\right) \\
& \log \left(\frac{p_{i}}{1-p_{i}}\right)=\beta_{0}+\beta_{1} X_{1 i}+\cdots+\beta_{k} X_{k i}+\gamma \text { Autocovaraite }_{i} \\
& \text { Autocovariate }_{i}=\frac{\sum_{j=1}^{n_{i}} w_{i j} y_{i j}}{\sum_{j=1}^{n_{i}} w_{i j}} \\
& w_{i j}=\frac{1}{d_{i j}}
\end{aligned}
$$

Where, $i=1, \ldots, 1222$, is the index of the patient; $Y_{\mathrm{i}}$ is outcome variable corresponding to the $i t h$ patient, 1 if dead, 0 if alive; $p_{i}$ is the probability of $Y_{\mathrm{i}}$ being a die; $\mathrm{X}_{1 \mathrm{i}}, \ldots, X_{m i}$ are the covariates corresponding to the ith patient; $\beta_{0}, \ldots, \beta_{m}$ are the regression coefficients of covariates; $\gamma$ is the regression coefficient of Autocovariate $e_{\text {that }}$ is the autocovariate of the ith patient to represent spatial correlations effects; $n_{i}$ is the number of neighbors of patient $i$; $w_{i j}$ is the spatial weight between patient $i$ and patient $j$, equal to the inverse of Euclidean distance $\left(d_{i j}\right)$ between them. 


\section{Variable Selection and Model Development}

Variable selection is made using backward logistic regression models and recorded the Akaike Information Criterion (AIC) of each fitted model (27). First, a full model was built containing all variables; to remove or select a variable, this model's AIC value with and without the variable is compared; if their difference is not greater than two, that variable is retained in the model.

Secondly, Moran's I statistic was used to check the spatial autocorrelation of residuals of the final ordinary logistic model. Moran's I is an index whose value is in the range from approximately -1 to 1 . Positive signage indicates positive spatial autocorrelation, while negative signage indicates negative spatial autocorrelation - a value of zero representing no spatial autocorrelation (28).

Thirdly, to account for spatial autocorrelation, autocovariate was calculated using the autocov_dist function in the "spdep" R package (29). The autologistic model was built with autocovariate as an additional independent variable in the final ordinal logistic model. Finally, residuals of the autologistic model were assessed by Moran's I test to ensure that the inclusion of autocovariates led to residual's independence, and there was no spatial autocorrelation. All analyses were performed using SPSS version 16 and $\mathrm{R}$ statistical software version 4.0.0 at the significant level of 0.05 .

\section{Ethics Statement:}

The protocol was approved by the Ethics Committee of NIMAD (IR.NIMAD.REC.1397.078) and also MUMS (Code: IR.MUMS.REC.1399.459). Data collection was also accomplished by the official authorization from Mashhad University of Medical Sciences (MUMS), Iran (research project number: 981153). It was performed in accordance with the ethical standards of the Declaration of Helsinki. Given the retrospective design, the informed consent was waived by the Ethics Committee of NIMAD (National Institute for Medical Reseach Development).

\section{Results}

\section{Description of the population}

One thousand two hundred and twenty-two patients with suspected stroke were included in the study which $14.2 \%$ (174 patients) died in hospital. A total of $608(50.2 \%)$ were males with a mean age of $69.87 \pm 13.61$ and $608(49.8 \%)$ females with a mean age of $70.14 \pm 13.94,806$ 
(67.4\%) cases had hypertension. The majority of patients $(84.1 \%)$ lived inside the city boundary. The median length of stay was $3(\mathrm{IQR}=6)$. Nine-hundred forty patients $(76.9 \%)$ were discharged within the first week after admission. Table 1 summarizes the demographic and EMS calls characteristics of suspected stroke patients. There was no significant difference between male and female in-hospital mortality ( $46 \%$ vs. 54\%, p=0.22). Two hundred ninety-six patients (24.2\%) were younger than or equal to 60 years, those who died were older (mean age=73.5 vs. $69.4, \mathrm{p}<0.001)$. The mean delay time of patients who died significantly was longer than patients who did not die $(\mathrm{p}=0.04)$. The median length of stay in the patients who died during the same hospitalization was significantly longer than that of the surviving population $(p<0.001)$. The results indicate no significant difference between those who died and those who survived in terms of mean response time, transport time, and distance to the hospital ( $p>0.05)$. However, the accessibility rate of an ambulance was significant $(\mathrm{p}=0.03)$.

Table 1: Demographic and clinical characteristics of suspected stroke patients, Mashhad, 2018

\begin{tabular}{|c|c|c|c|c|}
\hline & \multirow{2}{*}{$\begin{array}{c}\text { Total } \\
(n=1222)\end{array}$} & \multicolumn{2}{|c|}{ Died } & \multirow[b]{2}{*}{ P-value } \\
\hline & & $\begin{array}{c}\text { Yes } \\
(\mathbf{n}=174)\end{array}$ & $\begin{array}{c}\text { No } \\
(n=1048)\end{array}$ & \\
\hline \multicolumn{5}{|l|}{ Sex, n $(\%)$} \\
\hline Male & $614(50.2)$ & $80(46.0)$ & $534(51.0)$ & \multirow{2}{*}{0.22} \\
\hline Female & $608(49.8)$ & $94(54.0)$ & $514(49.0)$ & \\
\hline \multicolumn{5}{|l|}{ Age, n (\%) } \\
\hline$\leq 60$ & $296(24.2)$ & $27(15.5)$ & $269(25.7)$ & \multirow{2}{*}{0.004} \\
\hline$>60$ & $926(75.8)$ & $147(84.5)$ & $779(74.3)$ & \\
\hline Age, Mean \pm SD & $70.5 \pm 13.6$ & $73.5 \pm 13.7$ & $69.4 \pm 13.6$ & $<0.001$ \\
\hline \multicolumn{5}{|l|}{ Hypertension, n (\%) } \\
\hline Yes & $806(67.4)$ & $107(63.3)$ & $699(68.1)$ & \multirow[t]{2}{*}{0.22} \\
\hline No & $390(32.6)$ & $62(36.7)$ & $328(31.9)$ & \\
\hline \multicolumn{5}{|l|}{ Residency, n (\%) } \\
\hline Urban area & $1028(84.1)$ & $146(83.9)$ & $882(84.2)$ & \multirow{2}{*}{0.93} \\
\hline Suburbia & $194(15.9)$ & $28(16.1)$ & $166(15.8)$ & \\
\hline $\begin{array}{l}\text { Accessibility rate of an ambulance(per } 1 \text { million } \\
\text { inhabitants) (Mean } \pm \text { SD) }\end{array}$ & $27.3 \pm 6.7$ & $26.3 \pm 7.2$ & $27.7 \pm 6.8$ & 0.03 \\
\hline Delay time $($ Sec $)($ Mean \pm SD $)$ & $37.7 \pm 31.0$ & $42.0 \pm 37.0$ & $36.9 \pm 30.0$ & 0.04 \\
\hline Response time (Min) $($ Mean \pm SD) & $9.0 \pm 4.0$ & $9.1 \pm 3.9$ & $9.0 \pm 4.2$ & 0.8 \\
\hline Transport time $($ Min $)($ Mean \pm SD $)$ & $21.8 \pm 12.2$ & $22.4 \pm 14.3$ & $21.7 \pm 11.7$ & 0.4 \\
\hline Screening time (hour) & $0.25 \pm 0.3$ & $0.3 \pm 0.3$ & $0.2 \pm 0.1$ & $<0.001$ \\
\hline Distance to hospital $(\mathrm{km})($ Mean \pm SD $)$ & $8.1 \pm 4.2$ & $8.2 \pm 4.9$ & $8.1 \pm 4.0$ & 0.9 \\
\hline Length of stay, median(IQR) & $3(6.0)$ & $6.5(10.0)$ & $2(6.0)$ & $<0.001$ \\
\hline Length of stay, n (\%) & & & & \\
\hline
\end{tabular}




\begin{tabular}{lllll}
$\leq 7$ & $940(76.9)$ & $95(54.6)$ & $845(80.6)$ & $<0.001$ \\
$>7$ & $282(23.1)$ & $79(45.4)$ & $203(19.4)$ & \\
\hline
\end{tabular}

Despite the lack of a significant effect of sex on mortality in the univariate analysis, prior research indicates that mortality predictors may differ according to sex $(10,23,30,31)$. Table 2 shows the demographic and clinical features according to mortality stratified by sex. In both sexes, age and length of stay were associated with higher mortality $(\mathrm{p}<0.05)$. Also, for males, distance to a hospital was associated with higher mortality $(\mathrm{p}=0.03)$.

Table 2: Univariate analysis of the association between risk factors and in-hospital stroke mortality, stratified by sex, Mashhad, 2018

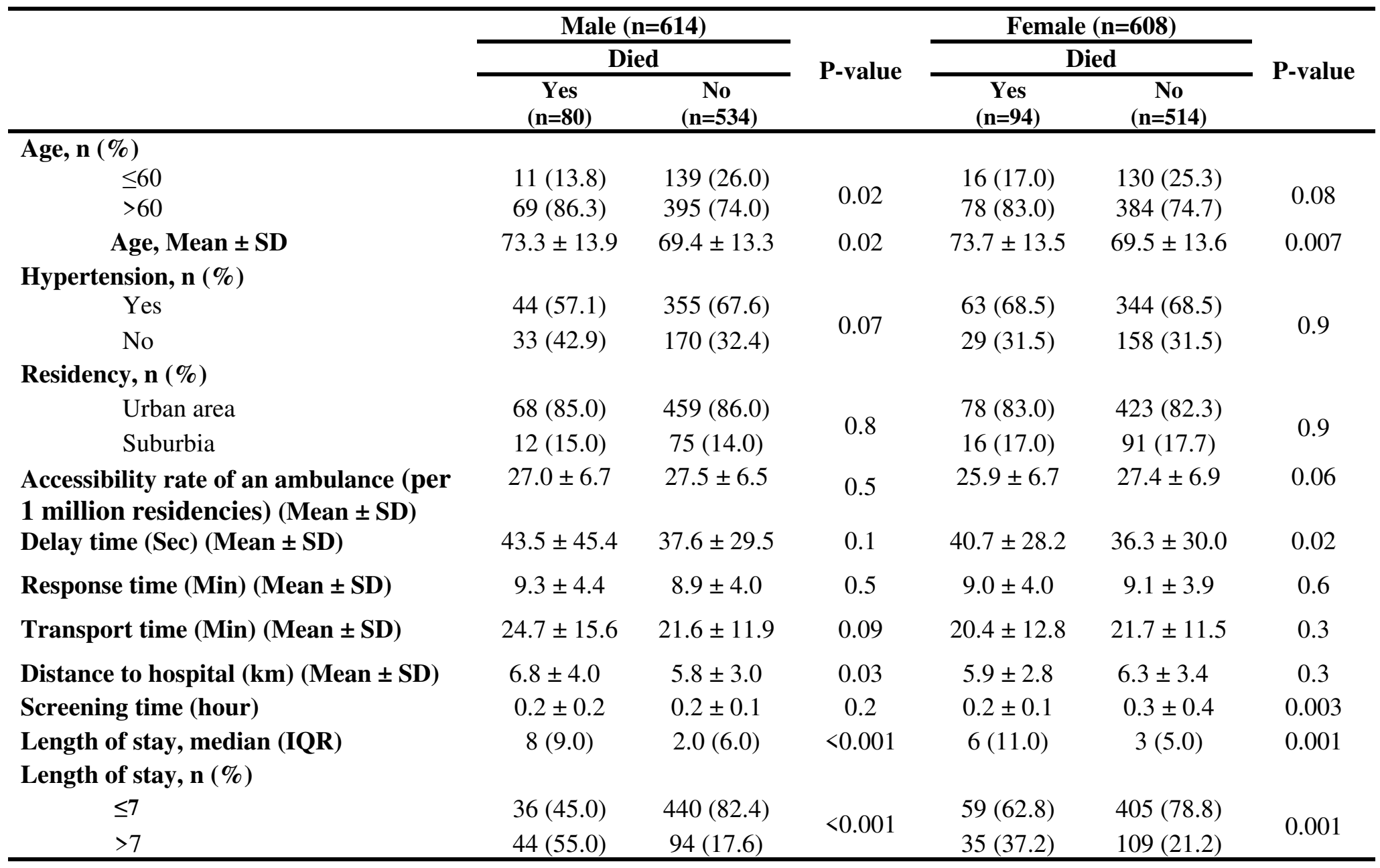

Autologistic modeling 
Six independent variables were selected in backward AIC variable selection, and the final logistic model is included age, sex, accessibility rate of an ambulance (per 1 million), delay time (Sec), Screening time (hour), length of stay (day). The Moran's I test indicated a significant spatial autocorrelation in the final ordinary logistic model's residuals. This means that models incorporating a spatial dependence component should be most appropriate. Autologistic regression was applied based on these six significant variables and autocovariate term, which the ordinary logistic model computed. The parameters and associated $95 \%$ confidence intervals of the spatial autologistic model are given in Table 3.

The autologistic regression model results showed that age (OR=1.03, 95\% CI 1.02 to 1.04), accessibility rate of an ambulance ( $\mathrm{OR}=0.97,95 \% \mathrm{CI} 0.95$ to 0.99$)$ are significant risk factors that are positively related to the occurrence of in-hospital mortality in suspected strokes. However, screening time is negatively associated with higher in-hospital mortality. So that, per every one hour increase in screening time, the odds ratio of in-hospital 0.15 decreased (95\% CI 0.03 to 0.58 ) (Table 3 ).

Different univariate analysis results led us to perform separate predictive models stratified by sex. the autologistic regression model revealed that age has a positive association with inhospital mortality. Per everyone increase in the age by adjusting for the effects of other variables, the odds of in-hospital mortality of suspected stroke patients had 3\% (overall and in men) and $2 \%$ (in women) increase. In-hospital mortality decreased with each additional accessibility rate of an ambulance, with an odds ratio of 0.97 per unit (95\% CI 0.95 to 0.99 ). However, there was no significant association between delay time and stroke mortality $(\mathrm{OR}=1.003,95 \% \mathrm{CI} 0.99$ to 1.008).

Table 3: Risk factors related to in-hospital mortality in suspected stroke patients, autologistic regression model overall and stratified by sex

\begin{tabular}{|c|c|c|c|}
\hline & \multirow[t]{2}{*}{ Total } & \multicolumn{2}{|c|}{ Sex } \\
\hline & & Men & Women \\
\hline & OR $(95 \% \mathrm{CI})$ & OR $(95 \%$ CI $)$ & OR $(95 \% \mathrm{CI})$ \\
\hline \multicolumn{4}{|l|}{ Sex } \\
\hline Male & $1.3(0.92-1.84)$ & - & - \\
\hline Female & Ref & - & - \\
\hline Age & $1.03(1.02-1.04)$ & $1.03(1.01-1.05)$ & $1.02(1.003-1.04)$ \\
\hline \multicolumn{4}{|l|}{ Hypertension } \\
\hline Yes & $1.17(0.81-1.67)$ & $1.35(0.80-2.29)$ & $1.01(0.60-1.65)$ \\
\hline
\end{tabular}


No

Accessibility rate of ambulance

(per 1 million residency)

delay time (Sec)

Screening time (hour)

Length of stay

Autocovariate
Ref

\begin{tabular}{lll}
$0.97(0.95-0.99)$ & $0.98(0.94-1.02)$ & $0.96(0.93-0.99)$ \\
& & \\
$1.003(0.99-1.008)$ & $1.002(0.99-1.009)$ & $1.003(0.99-1.009)$ \\
$0.15(0.03-0.58)$ & $0.44(0.55-1.72)$ & $0.05(0.004-0.47)$ \\
$1.03(1.02-1.04)$ & $1.04(1.02-1.06)$ & $1.03(1.01-1.04)$ \\
$5.3(0.01-1474.1)$ & $0.0012(0.0007-0.01)$ & $318(2.23-655)$ \\
\hline
\end{tabular}

The Moran's I statistic results showed no spatial autocorrelation in the residuals from the autologistic regression model. The autologistic regression model is preferred over the ordinary logistic regression model because it could cover spatial autocorrelations in the in-hospital mortality of suspected stroke patients. Then, ROC analysis was used for model evaluation. The area under the curve (AUC) was obtained as $70.1 \%(65.0 \%-73.5 \%)$, indicating the selected model's adequate predictive ability.

Figure 1 maps the estimated values of the autocovariate variable introduced in equation (3). The larger values of the autocovariate variable indicate neighborhoods with higher odds of in-hospital stroke mortality. We understood that there was a remarkable geographical variation in in-hospital stroke mortality in Mashhad neighborhoods, in a way that some neighborhoods in the suburban areas and northeastern parts of Mashhad had a higher odds of in-hospital stroke mortality.

In the north-east of the study area, the difference is more notable due to the socio-economic deprivations such as having low-income occupations, lower availability, quality of primary care, and less education. Also, other few neighborhoods have high odds of in-hospital mortality, which could be due to having more pensioners or pre-retirees and an older population. 


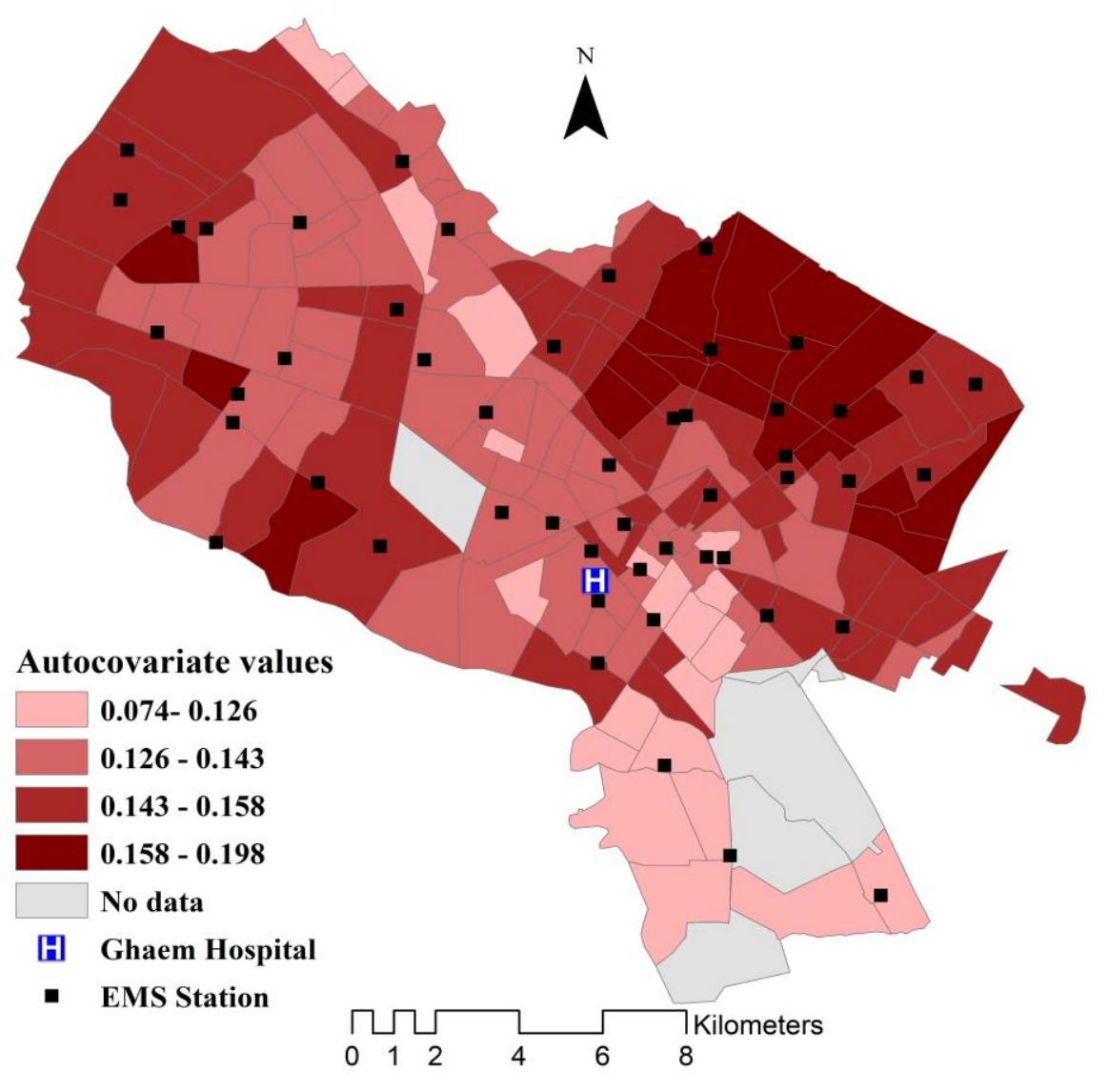

Figure 1. The spatial distribution of the autocorrelation term: the autocovariate variable in the neighborhood, Mashhad

\section{Discussion}

This study's objective was to investigate possible disparities in in-hospital mortality and its determinants among suspected stroke patients transferred by EMS in Mashhad, Iran. This study highlighted spatial variations of in-hospital mortality and its important contributing factors among suspected stroke patients in the emergency department of Mashhad, Iran.

In this study, the overall in-hospital stroke mortality rate was $14.2 \%$ higher than previous global studies $(23,32,33)$. However, it's lower than the mortality rate in some countries (34) and other 
Iran areas $(8,9,35)$. The in-hospital stroke mortality rates vary between countries and areas related to incidence rate, case fatality rate (36).

The ordinary regression model assumes that the dependent and explanatory variables' relationship is a stationary spatial process. The advantage of this study compared to existing studies is to account for spatial correlations in modeling. The autologistic regression model is a spatial model that considers the spatial residual autocorrelation effect in the model and reduces the contribution of the residual significantly by introducing a spatial autocorrelation variable. We The autologistic regression model revealed that the most relevant independent factor associated with in-hospital stroke mortality were age, length of stay in overall cases, in men and women, accessibility rate of an ambulance, screening time. The autocovariate in men and women was significant and showed higher odds of mortality in the suburb and northeastern areas. Several factors may have contributed to this result; for example, northeastern neighborhoods of Mashhad have a very high population density but low health facilities. In other words, in Mashhad, the focus of health centers is in the city's central neighborhoods, and these areas of the city have higher access to health services, and the surrounding areas have lower access to health services $(37,38)$. Therefore, health policymakers should pay attention to inequalities in healthcare resources' spatial distribution. Another reason for the higher probability of mortality in suburbs and northeastern areas is socio-economic deprivations. In Mashhad, the neighborhoods are not evenly distributed in terms of socio-economic status. There are clusters of urban poverty that have focused in the eastern part and marginal neighborhoods in Mashhad (39).

In this study, most stroke patients $(75.8 \%)$ were aged older than 60 . our findings indicate that age represents an important independent factor of death so that overall, each additional year is associated with a 3\% relative increase in in-hospital stroke mortality. Studies indicated an increased probability of in-hospital stroke mortality is associated with increasing age (40-42). The average length of stay was 6.52 days, and $23.1 \%$ of patients hospitalized more than one week also, the mean length of stay in the patients who died during the hospitalization was longer than other patients. The model results indicated that in-hospital mortality was associated with longer length of stay, and for everyday increase length of stay, the odds of in-hospital mortality $3 \%$ increased. Previous studies show a strong positive correlation between in-hospital stroke mortality and long length of stay $(8,23,43)$.

In 2018 mean accessibility rate of an ambulance in Mashhad city was 27 (per 1 million inhabitants) (25). Our findings indicate the accessibility rate of an ambulance is negatively 
associated with in-hospital mortality. With an increase in the accessibility rate of an ambulance, the odds of in-hospital stroke mortality decrease. Previous studies have shown more access to EMS increased the likelihood of therapy with rt-PA and decreased visiting time by an emergency physician, time to CT, and visiting time by a neurologist (44-46). Our results revealed that EMS's access rate in the suburban areas was lower than in central urban areas due to the huge aggregation of EMS stations in central areas. Patients in suburban areas with stroke were less likely to receive timely therapy and had higher in-hospital mortality than central areas (47). Therefore, health policymakers should pay attention to EMS stations' balanced distribution, especially in areas with high gaps and low EMS access rates.

Our study has some limitations. First, we only integrated data obtained from the EMS system database of City Emergency Management Center with patient medical records in the Ghaem Hospital. Patients who came to the hospital by personal vehicle were not considered. Second, our study was based on data acquired in a year and may differ from in-hospital stroke mortality patterns in multi-year data-based studies.

\section{Conclusion}

In Mashhad, the odds of in-hospital stroke mortality in the city's suburbs were higher than in central neighborhoods. Results autologistic regression model highlighted the direct association between age, accessibility rate of an ambulance, screening time and length of stay, and inhospital stroke mortality. Our results also showed disparities in access rate to EMS in Mashhad, and the prognosis of in-hospital stroke mortality could be improved by reducing delay time and increasing the access rate to EMS. These findings might help researchers and local health policymakers reduce health services disparities and in-hospital stroke mortality.

\section{Acknowledgments}

We want to thank NIMAD and the Vice-Chancellor of MUMS for their support and the medical stroke department in Ghaem Hospital, Mashhad, to provide access to data for this study.

\section{Authors' contributions}

Mohammad Taghi Shakeri: Conceptualization, Methodology, Supervision. Payam Sasannezhad: Data curation, contributions to the conception, review \& editing. Isa Nazar: Formal analysis, 
interperting, Writing and editing. Azadeh Saki: Investigation, Writing - review \& editing. Razieh Yousefi: Investigation, Writing - review \& editing. Ali Hadianfar: Formal analysis, Writing and drafting the manuscript, Visualization. Zahra jafari: data cleaning and preparation. All authors reviewed the manuscript.

\section{Funding}

The author(s) received no financial support for the research, authorship and/or publication of this article.

\section{Declaration of Conflicting Interests}

The author(s) declare that there is no conflict of interest.

\section{Availability of data and materials}

The datasets generated and/or analysed during the current study are not publicly available due [their containing information] but are available from the corresponding author on reasonable request.

\section{Consent for publication}

Not applicable

\section{Ethics approval and consent to participate}

Informed consent of the patients was deemed not necessary because of the retrospective design, and therefor exemption of ethical approval was granted by the Ethics Committee of NIMAD (National Institute for Medical Reseach Development).

\section{References}

1. Johnson CO, Nguyen M, Roth GA, Nichols E, Alam T, Abate D, et al. Global, regional, and national burden of stroke, 1990-2016: a systematic analysis for the Global Burden of Disease Study 2016. Lancet Neurol. 2019;18(5):439-58. 
2. Mousavi-Mirzaei SM, Talebi A, Amirabadizadeh A, Nakhaee S, Azarkar G, Mehrpour O. Increasing the Risk of Stroke by Opium Addiction. J Stroke Cerebrovasc Dis. 2019 Jul;28(7):1930-1935.

3. Mousavi-Mirzaei SM, Khorasani EY, Amirabadizadeh A, Nakhaee S, Baharshahi A, Rajabpour-Sanati A, Talebi A, Lamarine RJ, Mehrpour M, Mehrpour O. Comparison of blood lead concentrations in patients with acute ischemic stroke and healthy subjects. J Trace El.

4. Johnson W, Onuma O, Owolabi M, Sachdev S. Stroke: a global response is needed. Bull World Health Organ. 2016;94(9):634.

5. Mehrpour, M.; Yousefi, M.; AfzalAghaee, M.; Rakhshandeh, H.; Azizi, H.; Hadianfar, A.; Mehrpur, O.; Ghandehari, K.; Bahrami-Taghanaki, H. Evaluation of Dorema ammoniacum and Acupuncture' s Therapeutic Effects in Patients with Ischemic Stroke: A Randomized C.

6. Feigin VL. Stroke epidemiology in the developing world. Lancet (London, England). 2005;365(9478):2160-1.

7. Johnston SC, Mendis S, Mathers CD. Global variation in stroke burden and mortality: estimates from monitoring, surveillance, and modelling. Lancet Neurol. 2009;8(4):345-54.

8. Farhoudi M, Mehrvar K, Sadeghi-Bazargani H, Hashemilar M, Seyedi-Vafaee M, Sadeghi-Hokmabad E, et al. Stroke subtypes, risk factors and mortality rate in northwest of Iran. Iran J Neurol. 2017;16(3):112.

9. Borhani-Haghighi A, Safari R, Heydari ST, Soleimani F, Sharifian M, Kashkuli SY, et al. Hospital mortality associated with stroke in southern Iran. Iran J Med Sci. 2013;38(4):314.

10. Heuschmann PU, Kolominsky-Rabas PL, Misselwitz B, Hermanek P, Leffmann C, Janzen RWC, et al. Predictors of in-hospital mortality and attributable risks of death after ischemic stroke: the German Stroke Registers Study Group. Arch Intern Med. 2004;164(16):1761-8. 
11. Feigin VL, Lawes CMM, Bennett DA, Barker-Collo SL, Parag V. Worldwide stroke incidence and early case fatality reported in 56 population-based studies: a systematic review. Lancet Neurol. 2009;8(4):355-69.

12. Roth EJ, Lovell L, Harvey RL, Heinemann AW, Semik P, Diaz S. Incidence of and risk factors for medical complications during stroke rehabilitation. Stroke. 2001;32(2):523-9.

13. Thomas J, Saw KL, Adie K. Stroke mortality audit using the Structured Judgement Review method. Clin Med (Northfield Il). 2019;19(2):185.

14. Patel MD, Brice JH, Moss C, Suchindran CM, Evenson KR, Rose KM, et al. An evaluation of emergency medical services stroke protocols and scene times. Prehospital Emerg Care. 2014;18(1):15-21.

15. Saver JL. The 2012 Feinberg Lecture: treatment swift and treatment sure. Stroke. 2013;44(1):270-7.

16. Evenson KR, Foraker RE, Morris DL, Rosamond WD. A comprehensive review of prehospital and in-hospital delay times in acute stroke care. Int J Stroke. 2009;4(3):187-99.

17. Meurer WJ, Levine DA, Kerber KA, Zahuranec DB, Burke J, Baek J, et al. Neighborhood influences on emergency medical services use for acute stroke: a populationbased cross-sectional study. Ann Emerg Med. 2016;67(3):341-8.

18. Brown AF, Liang L-J, Vassar SD, Merkin SS, Longstreth WT, Ovbiagele B, et al. Neighborhood socioeconomic disadvantage and mortality after stroke. Neurology. 2013;80(6):520-7.

19. Pedigo A, Aldrich T. Neighborhood disparities in stroke and myocardial infarction mortality: a GIS and spatial scan statistics approach. BMC Public Health. 2011;11(1):1-13.

20. Osypuk TL, Ehntholt A, Moon JR, Gilsanz P, Glymour MM. Neighborhood differences in post-stroke mortality. Circ Cardiovasc Qual Outcomes. 2017;10(2):e002547.

21. Besag J. Spatial interaction and the statistical analysis of lattice systems. J R Stat Soc Ser B. 1974;36(2):192-225. 
22. Ho W-M, Lin J-R, Wang H-H, Liou C-W, Chang K-C, Lee J-D, et al. Prediction of inhospital stroke mortality in critical care unit. Springerplus. 2016;5(1):1-9.

23. Kortazar-Zubizarreta I, Pinedo-Brochado A, Azkune-Calle I, Aguirre-Larracoechea U, Gomez-Beldarrain M, Garcia-Monco JC. Predictors of in-hospital mortality after ischemic stroke: A prospective, single-center study. Heal Sci reports. 2019;2(4):e110.

24. SCI (2016) Official report of statistical survey of population in Mashhad city archived by the Statistical Center of Iran. http://www.amar.org.ir. Accessed 2016.

25. Hashtarkhani S, Kiani B, Bergquist R, Bagheri N, VafaeiNejad R, Tara M. An age-integrated approach to improve measurement of potential spatial accessibility to emergency medical services for urban areas. Int J Health Plann Manage. 2020;35(3):788-98.

26. Anselin L. Local indicators of spatial association-LISA. Geogr Anal. 1995;27(2):93115.

27. Akaike H. A new look at the statistical model identification. IEEE Trans Automat Contr. 1974;19(6):716-23.

28. Cliff AD, Ord JK. Spatial processes: models \& applications. Taylor \& Francis; 1981.

29. Bivand R, Altman M, Anselin L, Assunção R, Berke O, Bernat A, et al. Package 'spdep'. https://cranr-projectorg/web/packages/pdep/spdeppdf Accessed 10 Feb 2017. 2016.

30. Reeves MJ, Bushnell CD, Howard G, Gargano JW, Duncan PW, Lynch G, et al. Sex differences in stroke: epidemiology, clinical presentation, medical care, and outcomes. Lancet Neurol. 2008;7(10):915-26.

31. Appelros P, Stegmayr B, Terént A. Sex differences in stroke epidemiology: a systematic review. Stroke. 2009;40(4):1082-90.

32. Bustamante A, Garcia-Berrocoso T, Rodriguez N, Llombart V, Ribo M, Molina C, et al. Ischemic stroke outcome: a review of the influence of post-stroke complications within the different scenarios of stroke care. Eur J Intern Med. 2016;29:9-21. 
33. Gebreyohannes EA, Bhagavathula AS, Abebe TB, Seid MA, Haile KT. In-hospital mortality among ischemic stroke patients in Gondar University Hospital: a retrospective cohort study. Stroke Res Treat. 2019;2019.

34. Sweileh WM, Sawalha AF, Al-Aqad SM, Zyoud SH, Al-Jabi SW. Predictors of inHospital Mortality after Acute Stroke: Impact of Gender. Int J Clin Exp Med [Internet]. 2009/01/30. 2009;2(1):41-7. Available from: https://pubmed.ncbi.nlm.nih.gov/19436831

35. stroke.

36. Goulart AC, Bensenor IM, Fernandes TG, Alencar AP, Fedeli LM, Lotufo PA. Early and one-year stroke case fatality in Sao Paulo, Brazil: applying the World Health Organization's stroke STEPS. J Stroke Cerebrovasc Dis. 2012;21(8):832-8.

37. Bazargan M. A case study on accessibility of medical and healthcare facilities in Mashhad using GIS. SAUES J [Internet]. 2018;1(1):39-48. Available from: http://www.sauesjournal.net/article_27593.html

38. Rahnama M rahim, Amirfakhriyan M. Analysis of spatial access to healthcare services in Mashhad city. Phys Sacial Plan [Internet]. 2013;1(3):61-74. Available from: http://psp.journals.pnu.ac.ir/article_2170.html

39. Farhadikhah H, Hataminejad H, Shahi A, Zafari M. Spatial Analysis of Urban Poverty in Neighborhood Levels (case study: Mashhad city). Urban Econ [Internet]. 2018;2(2):17-36. Available from: https://ue.ui.ac.ir/article_22724.html

40. Matsui H, Fushimi K, Yasunaga H. Variation in risk-standardized mortality of stroke among hospitals in Japan. PLoS One. 2015;10(10):e0139216.

41. Myint PK, O. Bachmann M, Loke YK, D. Musgrave S, Price GM, Hale R, et al. Important factors in predicting mortality outcome from stroke: findings from the Anglia Stroke Clinical Network Evaluation Study. Age Ageing. 2017;46(1):83-90.

42. Nimptsch U, Mansky T. Stroke unit care and trends of in-hospital mortality for stroke in G ermany 2005-2010. Int J Stroke. 2014;9(3):260-5. 
43. Lingsma HF, Bottle A, Middleton S, Kievit J, Steyerberg EW, Marang-Van De Mheen PJ. Evaluation of hospital outcomes: the relation between length-of-stay, readmission, and mortality in a large international administrative database. BMC Health Serv Res. 2018;18(1):110.

44. Investigators CASPR (CASPR). Prioritizing interventions to improve rates of thrombolysis for ischemic stroke. Neurology. 2005;64(4):654-9.

45. Schroeder EB, Rosamond WD, Morris DL, Evenson KR, Hinn AR. Determinants of use of emergency medical services in a population with stroke symptoms: the Second Delay in Accessing Stroke Healthcare (DASH II) Study. Stroke. 2000;31(11):2591-6.

46. Kleindorfer DO, Lindsell CJ, Broderick JP, Flaherty ML, Woo D, Ewing I, et al. Community socioeconomic status and prehospital times in acute stroke and transient ischemic attack: do poorer patients have longer delays from 911 call to the emergency department? Stroke. 2006;37(6):1508-13.

47. Hammond G, Luke AA, Elson L, Towfighi A, Joynt Maddox KE. Urban-Rural Inequities in Acute Stroke Care and In-Hospital Mortality. Stroke. 2020;51(7):2131-8. 


\section{Figures}

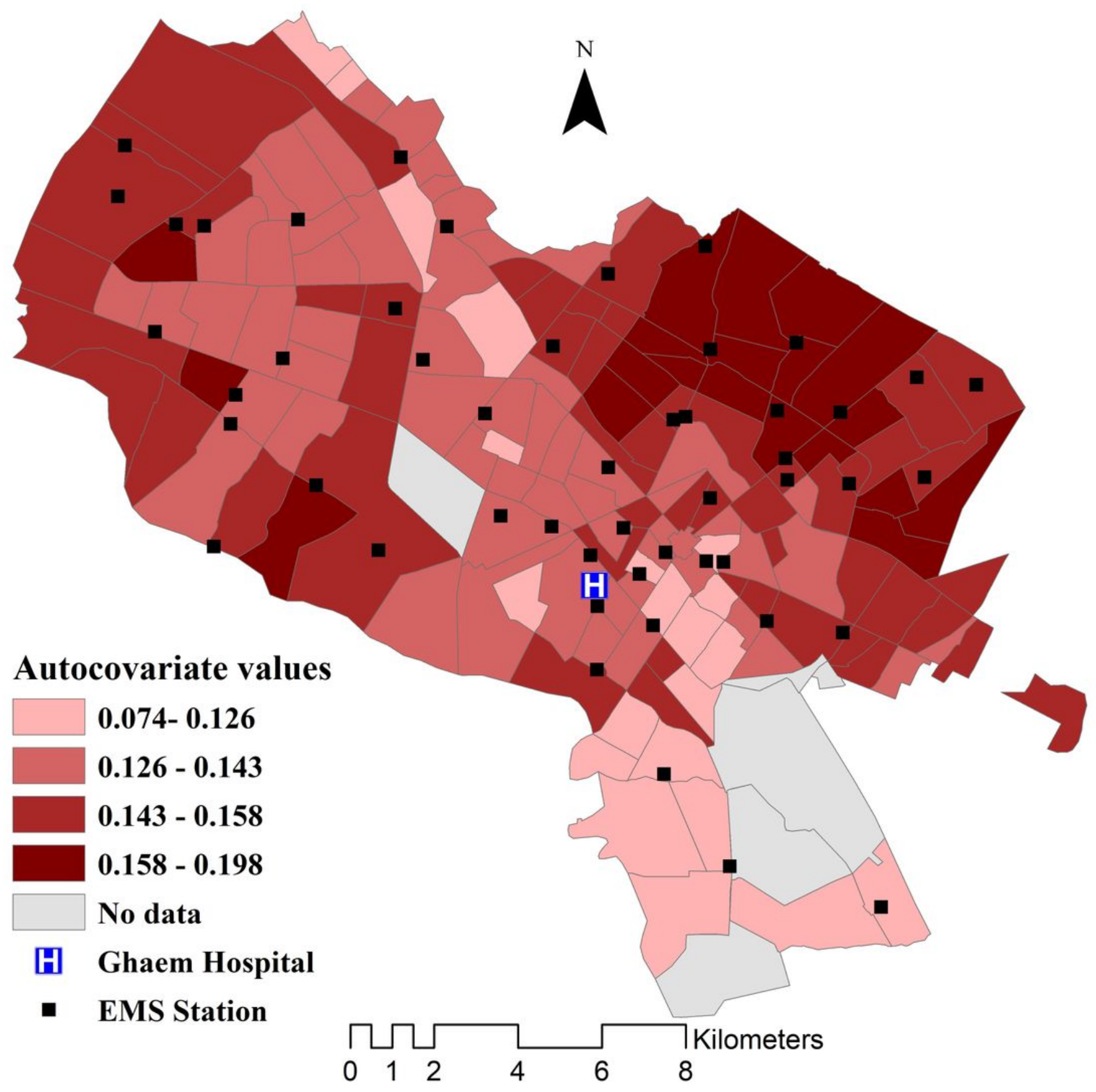

Figure 1

The spatial distribution of the autocorrelation term: the autocovariate variable in the neighborhood, Mashhad Note: The designations employed and the presentation of the material on this map do not imply the expression of any opinion whatsoever on the part of Research Square concerning the legal 
status of any country, territory, city or area or of its authorities, or concerning the delimitation of its frontiers or boundaries. This map has been provided by the authors. 